\title{
Three Sphere Inequality for Second Order Elliptic Equations with Coefficients with Jump Discontinuity
}

\author{
Michele Di Cristo and Yong Ren*
}

\begin{abstract}
This is a short note to complete the paper appeared in J. Differential Equations 261 (2016), no. 10, pp. 5306-5323, where a rough version of the classical well known Hadamard three-circle theorem for solution of an elliptic PDE in divergence form has been proved. Precisely, instead of circles, the authors obtain a similar inequality in a more complicated geometry. In this paper we clean the geometry and obtain a generalized version of the three-circle inequality for elliptic equation with coefficients with discontinuity of jump type.
\end{abstract}

\section{Introduction}

In this note we consider a generalization of the Hadamard three-circles theorem to solution of a divergence form elliptic equation in $\mathbb{R}^{n}$ with discontinuous coefficients. Motivated by the study of the inverse problem of determining an inclusion $D$ in an electrical conductor $\Omega$, the physical situation we aim to analyze is a layered medium, where each layer has a known conductivity, with a region $D$, whose conductivity is different from the surrounding material, located inside. Therefore, denoting by $A(x)$ the conductivity, $A$ turns out to be a piecewise constant function.

We are interested in obtaining a three spheres inequality of the form

$$
\|u\|_{L^{\infty}\left(B_{r_{2}}\right)} \leq C\|u\|_{L^{\infty}\left(B_{r_{1}}\right)}^{\tau}\|u\|_{L^{\infty}\left(B_{r_{3}}\right)}^{1-\tau}
$$

for solution $u$ of elliptic equation

$$
\operatorname{div}(A(x) \nabla u)=0, \quad \text { in } \Omega,
$$

*Politecnico di Milano, Italy (michele.dicristo@polimi.it, yong.ren@polimi.it). 
where $B_{r_{i}}, i=1,2,3$, is the ball of radius $r_{i}$ centered at any point $x \in \Omega \backslash D$, $0<r_{1}<r_{2}<r_{3}$ and $\tau \in(0,1)$.

This is a classical tool in PDEs that provides an estimate of the norm of the solution in a middle ball in term of its norm in a smaller ball and in a larger ball. This property, established by Hadamard for harmonic functions, has been obtained by Landis [La] for $L^{\infty}$-norms and Agmon for $L^{2}$-norms for solutions of general elliptic PDEs with smooth coefficients. Later refinements can be found in $[\mathrm{Ko}-\mathrm{Me}, \mathrm{Br}, \mathrm{Ku}]$. Recently the case with coefficients with jumps has been considered. In particular in [Fr-Li-Ve-Wa] a weaker version of (1.1) is obtained. Namely the authors prove a similar inequality for $L^{2}$ norms in a more complicated geometry instead of balls. A crucial tool to get this inequality is a suitable Carlemann estimate obtained in [DC-Fr-Li-Ve-Wa], where the second order elliptic operator considered has discontinuous coefficients with discontinuities that occur as jump at the interface. Let us mention here several closely related papers as [LR-Ro, LR-Ro2, LR-Le].

In this paper we proceed along this line refining the geometry of the inequality obtained in [Fr-Li-Ve-Wa] and getting a three sphere inequality.

These tools are important in application to inverse problems as they allow to evaluate quantitatively how some quantity propagates inside a domain. Specific applications can be found in [Fr-Li-Ve-Wa], where size estimates for unknown inclusions are proved, and in [DC-Re] where stability estimates for the inverse inclusion problem is studied.

In the next Section 2 we will state three sphere type theorem specifying the hypothesis needed. The proof is provided in Section 3 where the Carlemann estimate and the three region inequality used are recalled.

\section{Assumptions and Main Result}

In this Section we state our main result. We start by fixing some notations and listing the hypothesis we need. We denote by $\Omega$ a bounded open set in $\mathbb{R}^{n}$ with $C^{1, \alpha}$ boundary $\partial \Omega$ with constants $s_{0}, L_{0}$, where $0<\alpha \leq 1$, such that $|\Omega| \leq C r_{0}^{n}$, for some given $r_{0}>0$ with $C$ a positive constant. Assume that $\Sigma$ is a $C^{1,1}$ hypersurface with constants $s_{1}, L_{1}$ that divides $\Omega$ into two open sets $\Omega_{+}$and $\Omega_{-}$such that

$$
\Omega=\Omega_{+} \cup \Sigma \cup \Omega_{-}
$$

Denoting by $H_{ \pm}^{(\Omega)}=\chi_{\Omega_{ \pm}}$, we consider the conductivity equation

$$
\operatorname{div}(A \nabla u)=0, \quad \text { in } \Omega,
$$


where $A=H_{+}^{(\Omega)} A_{+}+H_{-}^{(\Omega)} A_{-}$with

$$
A_{ \pm}(x)=\left\{a_{i j}^{ \pm}(x)\right\}_{i, j=1}^{n}, \quad x \in \mathbb{R}^{n}
$$

a Lipschitz symmetric matrix-valued function satisfying for given constants $\lambda \in(0,1], \Lambda>0$

$$
\lambda|z|^{2} \leq A_{ \pm}(x) z \cdot z \leq \lambda^{-1}|z|^{2}, \quad \forall x \in \mathbb{R}^{n}, \forall z \in \mathbb{R}^{n}
$$

and

$$
\left|A_{ \pm}\left(x^{\prime}\right)-A_{ \pm}(x)\right| \leq \frac{\Lambda}{r_{0}}\left|x^{\prime}-x\right| .
$$

We can now state our main theorem.

Theorem 2.1. Let $u$ be a solution to (2.1) and $A_{ \pm}(x)$ satisfy (2.2) and (2.3). Then there exist $C>0$ depending on $\lambda, \Lambda, n$ such that

$$
\|u\|_{L^{\infty}\left(B_{l_{1} r}(z)\right)} \leq C\|u\|_{L^{\infty}\left(B_{r}(z)\right)}^{\tau}\|u\|_{L^{\infty}\left(B_{l_{2} r}(z)\right)}^{1-\tau},
$$

for $z \in \Omega \backslash D$, where $0<\tau<1$ depends on the a priori data and $1<l_{1}<l_{2}$ such that $B_{l_{2} r}(z) \subset \Omega$, for some $r<1$.

Remark 2.2. This result remain valid if we add lower order terms of the form $\sum_{ \pm} H_{ \pm}(W \nabla u+V u)$, where $W, V$ are bounded function, to (2.1). Its proof, indeed, makes use of an estimate that holds true for more general operators (see [Fr-Li-Ve-Wa, Remark 2.2]).

\section{Proof of Theorem 2.1}

In this section we provide the proof of Theorem 2.1. Without loss of generality, we can assume that the interface $\Sigma$ is planar. Indeed, since $\Sigma$ is $C^{1,1}$, for any $P \in \Sigma$ there exists a rigid transformation of coordinates under which $P=0$ and

$$
\Omega_{ \pm} \cap B_{r_{0}}(0)=\left\{(x, y) \in B_{r_{0}}(0) \subset \mathbb{R}^{n}: y \gtrless \psi(x)\right\},
$$

where $\psi$ is a $C^{1,1}$ function on $B_{r_{0}}^{\prime}(0) \subset \mathbb{R}^{n-1}$ satisfying $\psi(0)=0$ and $\|\psi\|_{C^{1,1}\left(B_{r_{0}}^{\prime}(0)\right)} \leq K_{0}$. Using the coordinate transform $\left(x^{\prime}, y^{\prime}\right)=T(x, y)=$ $(x, y-\psi(x))$ for $x \in B_{r_{0}}^{\prime}$, we reduce our analysis to the planar interface. Therefore we will prove Theorem 2.1 assuming $\Sigma$ to be planar. We denote by $H_{ \pm}=\chi_{\mathbb{R}_{ \pm}^{n}}$, where $\mathbb{R}_{ \pm}^{n}=\left\{(x, y) \in \mathbb{R}^{n-1} \times \mathbb{R}: y \gtrless 0\right\}$. Let $u_{ \pm} \in C^{\infty}\left(\mathbb{R}^{n}\right)$ and set

$$
u=H_{+} u_{+}+H_{-} u_{-}=\sum_{ \pm} H_{ \pm} u_{ \pm}
$$


we define

$$
\mathcal{L} u:=\sum_{ \pm} H_{ \pm} \operatorname{div}\left(A(x, y) \nabla u_{ \pm}\right) .
$$

To prove Theorem 2.1 we will make use of the following three-region inequality.

Theorem 3.1. Let $u$ be a solution of (3.1). There exist $C$ and $R$ depending on the a priori data such that if $0<R_{1}, R_{2}<R$, then

$$
\int_{U_{2}}|u|^{2} d x \leq C\left(\int_{U_{1}}|u|^{2} d x d y\right)^{\frac{R_{2}}{2 R_{1}+3 R_{2}}}\left(\int_{U_{3}}|u|^{2} d x d y\right)^{\frac{2 R_{1}+2 R_{2}}{2 R_{1}+3 R_{2}}},
$$

where

$$
\begin{aligned}
& U_{1}=\left\{-4 R_{2} \leq z(x, y), \quad \frac{R_{1}}{8 a}<y<\frac{R_{1}}{a}\right\} \\
& U_{2}=\left\{-R_{2} \leq z(x, y) \leq \frac{R_{1}}{2 a}, \quad y<\frac{R_{1}}{8 a}\right\} \\
& U_{3}=\left\{-4 R_{2} \leq z(x, y), \quad y<\frac{R_{1}}{a}\right\}
\end{aligned}
$$

$a=\alpha_{+} / \delta$ and

$$
z(x, y)=\frac{\alpha_{-}}{\delta} y+\frac{\beta}{2 \delta^{2}} y^{2}-\frac{1}{2 \delta}|x|^{2} .
$$

For the proof of this, we refer to [Fr-Li-Ve-Wa, Theorem 3.1]. Let us only mention that it is based on a proper use of a Carlemann estimate obtained in [DC-Fr-Li-Ve-Wa].

Let us now denote some parameters to describe the geometric properties of the regions. We use $l_{1}, l_{2}, l_{3}$ to represent the longest "length" for regions $U_{1}, U_{2}, U_{3}$ along $x$-axis. We use $d_{1}, d_{2}, d_{3}$ to represent the longest "depth" for regions $U_{1}, U_{2}, U_{3}$ along $y$-axis. With some calculations, we obtain

$$
\begin{aligned}
l_{1} & =l_{3}=2 \sqrt{\frac{\beta}{\delta}\left(\frac{R_{1}}{a}\right)^{2}+2 \alpha_{-} \frac{R_{1}}{a}+8 \delta R_{2}} \\
l_{2} & =2 \sqrt{\frac{\beta}{\delta}\left(\frac{R_{1}}{8 a}\right)^{2}+2 \alpha_{-} \frac{R_{1}}{8 a}+2 \delta R_{2}} \\
d_{1} & =\frac{7 R_{1}}{8 a} \\
d_{2} & =\frac{R_{1}}{8 a}+\frac{\delta}{\beta}\left(\alpha_{-}-\sqrt{\alpha_{-}^{2}-2 \beta R_{2}}\right) \\
d_{3} & =\frac{R_{1}}{a}+\frac{\delta}{\beta}\left(\alpha_{-}-\sqrt{\alpha_{-}^{2}-8 \beta R_{2}}\right)
\end{aligned}
$$


Proof of Theorem 2.1. For any point $O \in \Omega \backslash D$, we build a coordinator system $x$-O-y. First, we want to have $U_{1} \subset B_{r_{1}}$. Then, we will use a finite union of $U_{2}$ to cover $B_{r_{2}}$, that is, there exists $M<\infty$, such that $B_{r_{2}} \subset \cup_{j=1}^{M} U_{2 j}$. Finally, we want $\cup_{j=1}^{M} U_{3 j} \subset B_{r_{3}}$. All these can be done by choosing the proper $R_{1}, R_{2}, a$, i.e., the proper geometric structures for these regions.

(i) $U_{1} \subset B_{r_{1}}$. We want the longest distance between $O$ and any point in $U_{1}$ less then the radius of the $B_{r_{1}}$. In this case, it is easy to calculate $\left(\frac{l_{1}}{2}\right)^{2}+\left(\frac{R_{1}}{a}\right)^{2} \leq r_{1}^{2}$, which gives

$$
\left(\frac{\beta}{\delta}+1\right)\left(\frac{R_{1}}{a}\right)^{2}+2 \alpha_{-} \frac{R_{1}}{a}+8 \delta R_{2} \leq r_{1}^{2}
$$

(ii) $B_{r_{2}} \subset \cup_{j=1}^{M} U_{2 j}$. Since the Lebesgue measure of the whole domain $|\Omega|$ is finite. We can always cover $B_{r_{2}}$ by duplicating a finite amount of $U_{2 j}$, $j=1, \ldots, M$, along both $x$-axis and $y$-axis. In fact, we need at least $\frac{2 r_{2}}{l_{2}}$ amounts of $U_{2 j}$ along $x$-axis; and at least $\frac{2 r_{2}}{d_{2}}$ amounts of $U_{2 j}$ along the $y$-axis to cover the whole $B_{r_{2}}$. In this case, a wise choice of $M$ should be

$$
M=\left\lceil\frac{2 r_{2}}{l_{2}}\right\rceil \times\left\lceil\frac{2 r_{2}}{d_{2}}\right\rceil
$$

where $\lceil\cdot\rceil$ is the ceiling function, which maps any integer to the least integer that is greater or equal to itself.

(iii) $\cup_{j=1}^{M} U_{3 j} \subset B_{r_{3}}$. In the previous step, we use the union of $M$ regions. This will magnify the total "length" and "depth" of the union $\cup_{j=1}^{M} U_{3 j}$. We want the longest distance between $O$ and any point in $\cup_{j=1}^{M} U_{3 j}$ less than the radius of $B_{r_{3}}$. In this case, it is easy to calculate the total "length" of the union $\cup_{j=1}^{M} U_{3 j}$ is $l_{3}\left[\frac{2 r_{2}}{l_{2}}\right\rceil$; and the total "depth" of the union $\cup_{j=1}^{M} U_{3 j}$ is $d_{3}\left\lceil\frac{2 r_{2}}{d_{2}}\right\rceil$. Thus, the longest distance should be less than $r_{3}$, which is

$$
\left(l_{3}\left\lceil\frac{2 r_{2}}{l_{2}}\right\rceil\right)^{2}+\left(d_{3}\left\lceil\frac{2 r_{2}}{d_{2}}\right\rceil\right)^{2} \leq r_{3}^{2}
$$

Subject to regularities (3.5), (3.7), as well as the geometric relationships; we could apply the three-region inequalities and the standard bound for $L^{\infty}$ norm (see [Gi-Tr, Chapter 8]) 


$$
\begin{aligned}
\|u\|_{L^{\infty}\left(B_{r_{2}}\right)} & \leq C\|u\|_{L^{2}\left(B_{r_{2}}\right)} \leq C\|u\|_{L^{2}\left(\cup_{j=1}^{M} U_{2 j}\right)} \\
& \leq C M\|u\|_{L^{2}\left(U_{2}\right)} \leq C M\|u\|_{L^{2}\left(U_{1}\right)}^{\gamma}\|u\|_{L^{2}\left(U_{3}\right)}^{1-\gamma} \\
& \leq C\|u\|_{L^{2}\left(U_{1}\right)}^{\gamma}\|u\|_{\left.L^{2}\left(\cup_{j=1}^{M} U_{3 j}\right)\right)}^{1-\gamma} \\
& \leq C\|u\|_{L^{2}\left(B_{r_{1}}\right)}^{\gamma}\|u\|_{L^{2}\left(B_{r_{3}}\right)}^{1-\gamma} \\
& \leq C\|u\|_{L^{\infty}\left(B_{r_{1}}\right)}^{\tau}\|u\|_{L^{\infty}\left(B_{r_{3}}\right)}^{1-\tau}
\end{aligned}
$$

where $\|u\|_{L^{2}\left(B_{r}\right)}=r^{n} \int_{B_{r}}|u|^{2}$ and $C$ depends on $\lambda, \Lambda$

\section{References}

[Ag] S. Agmon, Unicitè et convexitè dans les problémes diffèrentiels, (French) Sèminaire de Mathématiques 13, Univ. de Montréal 1966.

[Br] R. Brummelhuis, Three-spheres theorem for second order elliptic equations, J. Anal. Math. 65 (1995), pp. 179-206.

[DC-Fr-Li-Ve-Wa] M. Di Cristo, E. Francini, C-L. Lin, S. Vessella, J-N. Wang, Carleman estimate for second order elliptic equations with Lipschitz leading coefficients and jumps at an interface, J. Math. Pures Appl. 108 (2017), pp. 163-206.

[DC-Re] M. Di Cristo, Y. Ren, Stable determination of an inclusion in a layered medium, Math. Methods Appl. Sci., in press.

[Fr-Li-Ve-Wa] E. Francini, C-L. Lin, S. Vessella, J-N. Wang, Three-region inequalities for the second order elliptic equation with discontinuous coefficients and size estimate, J. Differential Equations 261 (2016), pp. $5306-5323$.

[Ko-Me] J. Korevaar, J. Meyers, Logarithmic convexity for supremum norms of harmonic functions, Bull. London Math. Soc. 26 (1994), pp. 353-362.

[Ku] I. Kukavica, Quantitative uniqueness for second-order elliptic operators, Duke Math. J. 91 (1998), pp. 225-240.

[Gi-Tr] D. Gilbarg, N. Trudinger, Elliptic partial differential equations of second order, reprint of the 1998 edition, Classics in Mathematics, SpringerVerlag, Berlin (2001). 
[La] E. M. Landis, A three-spheres theorem, (Russian) Dokl. Akad. Nauk SSSR 148 (1963), pp. 277-279.

[LR-Le] J. Le Rousseau, N. Lerner, Carleman estimates for anisotropic elliptic operators with jumps at an interface, Anal. PDE 6 (2013), pp. 1601-1648.

[LR-Ro] J. Le Rousseau, L. Robbiano, Carleman estimate for elliptic operators with coefficients with jumps at an interface in arbitrary dimension and application to the null controllability of linear parabolic equations, Arch. Ration. Mech. Anal. 195 (2010), pp. 953-990.

[LR-Ro2] J. Le Rousseau, L. Robbiano, Local and global Carleman estimates for parabolic operators with coefficients with jumps at interfaces, Invent. Math. 183 (2011), pp. 245-336. 